\title{
Dihydroartemisinin inhibits the migration of esophageal cancer cells by inducing autophagy
}

\author{
XIAO $\mathrm{CHEN}^{1 *}$, LAN-YING HE${ }^{2 *}, \mathrm{SHU} \mathrm{LAI}^{3}$ and YAO HE \\ ${ }^{1}$ Department of Pharmacy, Chongqing Emergency Medical Center, Chongqing University Central Hospital, Chongqing \\ 400014; ${ }^{2}$ Department of Gastroenterology, Tumor Hospital Affiliated to Chongqing University, Chongqing 400030; \\ ${ }^{3}$ Department of Pharmacy, Jiulongpo District People's Hospital of Chongqing, Chongqing 400050, P.R. China
}

Received December 26, 2019; Accepted June 23, 2020

DOI: 10.3892/ol.2020.11955

\begin{abstract}
Esophageal cancer (EC) is a complex gastrointestinal malignancy and its global incidence rate ranks 7 th among all cancer types. Due to its aggressive nature and the potential for early metastasis, the survival rates of patients with EC are poor. Dihydroartemisinin (DHA) is the primary active derivative of artemisinin, and, as well as its use as an anti-malarial, DHA has also exhibited antitumor activity in various cancer models, such as cholangiocarcinoma, head and neck carcinoma, and hepatocellular carcinoma cells. However, the molecular mechanisms underlying the antitumor effect of DHA in the treatment of EC remains poorly understood. The results of the present study demonstrated that DHA significantly inhibited the migration of TE-1 and Eca-109 EC cells in a dose-dependent manner by activating autophagy. DHA treatment also significantly reversed epithelial-mesenchymal transition (EMT) by downregulating the EMT-associated markers, $\mathrm{N}$-cadherin and vimentin, and upregulating the expression of E-cadherin. Mechanistically, DHA treatment decreased Akt phosphorylation and inhibited the Akt/mTOR signaling pathway, leading to the activation of autophagy. The levels of the autophagy-associated proteins were suppressed and DHA-mediated inhibition of migration in EC cells was reversed when an active form of Akt was overexpressed. In conclusion, the present study demonstrated the potential value of DHA in the treatment of EC, and revealed the underlying mechanism by which FDHA inhibits cellular migration.
\end{abstract}

Correspondence to: Professor Yao He, Department of Pharmacy, Chongqing Emergency Medical Center, Chongqing University Central Hospital, 1 Jiankang Road, Yuzhong, Chongqing 400014, P.R. China E-mail: yaohe_6425@hotmail.com

Professor Shu Lai, Department of Pharmacy, Jiulongpo District People's Hospital of Chongqing, 5 Yejin Village, Yejin Road, Jiulongpo, Chongqing 400050, P.R. China

E-mail:672305465@qq.com

*Contributed equally

Key words: dihydroartemisinin, esophageal cancer, autophagy, migration, Akt

\section{Introduction}

Esophageal cancer (EC) is a complex and heterogeneous gastrointestinal malignancy, which is ranked 7th with respect to incidence rate and 6th for overall mortality rate in 2018 for 36 cancer types, leading to $>400,000$ deaths each year, globally (1). Esophageal adenocarcinoma (EADC) and esophageal squamous cell carcinoma (ESCC) are the most common subtypes of EC (2). Cases of ESCC are primarily reported in lower income regions, such as South-Eastern and Central Asia, and the global incidence rate for ESCC is higher compared with that in EADC (3-5). There are various different approaches to treating EC, such as chemotherapy, radiotherapy, surgery and a combination treatment; however, the prognosis of patients with EC is still a challenge. Developing effective chemoprevention and chemotherapeutics to treat EC is difficult, as its etiology varies from person to person, such as tobacco and alcohol in North America and betel quid in India (6). Inefficient treatments result in poor 5-year survival rate and, even following advanced radical esophagectomy, the 5-year survival rate of patients with EC in 2018 is still <20\% in China (7).

The primary cause of the low survival rate and poor prognosis for EC is early invasion and metastasis, which is a biological characteristic of cancer cells (8). The occurrence of invasion and metastasis involves complex multiple-step processes, numerous signaling pathways and regulation by transcription and growth factors, such as TGF- $\beta$, Snail, ZEB and bHLH (9-12), and the tumor microenvironment $(13,14)$. Epithelial-mesenchymal transition (EMT) is an important process during tumor cell development and metastasis, which involves the transformation of polar epithelial cells into active mesenchymal cells, which can migrate freely between the cell stroma (15). EMT is marked by the loss of epithelioid cell polarity and the acquisition of mesenchymal cell characteristics, with reduced expression of typical epithelial cell markers (such as E-cadherin) and increased mesenchymal cell markers (such as N-cadherin) (15). This phenotypic transformation releases tumor cells from intercellular connections, enabling non-invasive tumor cells to acquire invasive capacity, thereby promoting local invasion and distant tumor metastasis (16).

Numerous studies have indicated that EMT is regulated by autophagy, a critical cell survival process through which 
aging organelles and misfolded proteins are degraded $(17,18)$. Although previous studies $(19,20)$ showed that dihydroartemisinin (DHA), the major active metabolite of artemisinin, might have an antitumor activity in various cancer models, whether DHA could regulate the EMT and migration of EC cells remains unknown. In the present study, a series of experiments were conducted to investigate the migration inhibition and underlying molecular mechanisms of DHA. Exploring the molecular mechanism could provide new perspectives and therapeutic targets for clinical therapy in patients with EC.

\section{Materials and methods}

Cell culture and reagents. The TE-1 and Eca109 EC cell lines were purchased from the Library of Typical Culture of the Chinese Academy of Sciences. The cells were cultured in Dulbecco's modified Eagle's medium (DMEM; Gibco; Thermo Fisher Scientific, Inc.) containing $10 \%$ fetal bovine serum (Gibco; Thermo Fisher Scientific, Inc.) in a $37^{\circ} \mathrm{C}$ incubator $\left(95 \%\right.$ air and $5 \% \mathrm{CO}_{2}$ ). DHA was dissolved in DMSO (both Sigma-Aldrich; Merck KGaA) to a final concentration of $20 \mu \mathrm{g} / \mathrm{ml}$ and stored at $-20^{\circ} \mathrm{C}$, in the dark until further use.

Immunofluorescence assay. EC cells were harvested using $0.25 \%$ trypsin-EDTA (HyClone; Cytiva) and seeded into a 24 -well plate $\left(1 \times 10^{5}\right.$ cells/well) to adhere overnight. The cells were then transfected with $800 \mathrm{ng}$ pcDNA3.1-LC3-GFP, a plasmid expressing green fluorescent protein (GFP) and microtubule-associated protein 1A/1B-light chain 3 (LC3) (coding sequence of GFP and human LC3 were sub-cloned into pcDNA3.1(V790-20, Invitrogen; Thermo Fisher Scientific, Inc.), hereafter referred to as GFP-LC3) using Lipofectamine $^{\circledR} 2000$ (Invitrogen; Thermo Fisher Scientific, Inc.). The culture medium was replaced following $6 \mathrm{~h}$ of incubation at $37^{\circ} \mathrm{C}$ and $2 \mu \mathrm{g} / \mathrm{ml}$ DHA was added prior to incubation for a further $24 \mathrm{~h}$. The samples were fixed with $4 \%$ paraformaldehyde at room temperature for $15 \mathrm{~min}$ and permeabilized with $0.1 \%$ Triton-X 100 buffer for $10 \mathrm{~min}$, and the slides were subsequently stained with DAPI (Beyotime Institute of Biotechnology) for $5 \mathrm{~min}$ at room temperature.

Wound-healing assay. A total of $1 \times 10^{6}$ cells/well were seeded into a 6-well plate and cultured overnight to adhere. The cell monolayers were scratched with a $1-\mathrm{ml}$ pipette tip, and the culture medium was replaced with fresh medium containing $3 \%$ FBS to maintain cell survival. DHA was added at concentrations of 1,2 and $5 \mu \mathrm{g} / \mathrm{ml}$, and the cells were incubated for a further $24 \mathrm{~h}$ in a $37^{\circ} \mathrm{C}$ incubator (95\% air and $5 \% \mathrm{CO}_{2}$ ). Images were captured at 0 - and 24-h time points by fluorescence microscopy (magnification, $\mathrm{x} 100$ ), and the wound-closure area was calculated using ImageJ software v1.51d (National Institutes of Health).

Migration assay. The migration assay was performed using 24-well plates with Transwell inserts (filter membrane pore-size, $8-\mu \mathrm{m}$; Corning, Inc.). Briefly, $2 \times 10^{4}$ cells in FBS-free medium were added to the upper chambers of the Transwell inserts, while conditioned medium with $20 \%$ FBS was added to the lower chambers. DHA $(2 \mu \mathrm{g} / \mathrm{ml}), 3-\mathrm{MA}(2 \mathrm{mmol} / \mathrm{l}$; cat. no. HY-19312; MedChemExpress) or a combination of both were then added to the chambers, and the cells were incubated at $37^{\circ} \mathrm{C}$ for $24 \mathrm{~h}$. The non-migrated cells were removed with cotton swabs, and the cells on the underside of the filter membrane were washed with PBS and fixed with 4\% paraformaldehyde at room temperature for $15 \mathrm{~min}$. The chambers were subsequently stained with $0.1 \%$ crystal violet buffer for $30 \mathrm{~min}$ at room temperature, following washing with PBS. Migration was determined using the mean number of migratory cells from 20 visual fields with light microscope at x10 magnification.

Western blot analysis. A total of $1 \times 10^{6}$ cells/well were inoculated into a 6-well plate and left to adhere at $37^{\circ} \mathrm{C}$ overnight. The cells were treated with various concentrations of DHA $(1.25,2.5$ and $5.00 \mu \mathrm{g} / \mathrm{ml})$ or $2 \mathrm{mmol} / 13$-MA or a combined treatment for $24 \mathrm{~h}$, following removal of the culture medium. The cells were then lysed on ice with radioimmunoprecipitation assay lysis buffer (Sangon Biotech Co., Ltd.) and the supernatant was collected following centrifugation $(12,000 \times \mathrm{g}$ for $10 \mathrm{~min})$ at $4^{\circ} \mathrm{C}$. Protein concentration was determined using a bicinchoninic acid kit (Sangon Biotech Co., Ltd.). Equal amounts of protein (15 $\mu \mathrm{g}$ per well) were electrophoresed using $10 \%$ sodium dodecyl sulfate-polyacrylamide gels and transferred to PVDF membranes. The membranes were blocked with 1x EZ-Block A in PBS solution at $23^{\circ} \mathrm{C}$ for $1 \mathrm{~h}$ (Sangon Biotech Co., Ltd.). Subsequently, the membranes were incubated with LC3 (cat. no. L8918), sequestosome 1 (SQSTM1; cat. no. P0067), and $\beta$-actin antibodies (cat. no. SAB5600204) (all Sigma-Aldrich; Merck KGaA; dilution, 1:1,000), E-cadherin (cat. no. 3195S), $\mathrm{N}$-cadherin (cat. no. 13116S), vimentin (cat. no. 5741S), p-mTOR (cat. no. 5536S), mTOR (cat. no. 2983S), AKT (cat. no. 4685S), phosphorylated (p)-Akt (cat. no. 13038S) (all Cell Signaling Technology, Inc.; dilution, 1:1,000) for $12 \mathrm{~h}$ at $4^{\circ} \mathrm{C}$. Next, the membranes were incubated with HRP-labeled goat anti-rabbit IgG secondary antibodies (ZSGB-BIO; cat. no. ZB2301; dilution, 1:10,000) for $2 \mathrm{~h}$ at room temperature. The protein visualization was mediated by Western Blot Chemiluminescence HRP Substrate kit (Takara Bio, Inc.; cat. no. T7101A). The grey value was detected using ImageJ software v1.51d (National Institutes of Health).

Constitutively active (CA)-Akt overexpression assay. A total of $9 \times 10^{5}$ cells were seeded in 6 -well plates overnight, and $5 \mu \mathrm{g}$ CA-Akt plasmid (cat. no. 14751; Addgene, Inc.) and pcDNA3.1 empty vector (cat. no. V790-20, Invitrogen; Thermo Fisher Scientific, Inc.) was transfected into the cells using Lipofectamine ${ }^{\circledR} 3000$ (Invitrogen; Thermo Fisher Scientific, Inc.) when the cell fusion rate reached $85 \%$. The cell culture was replaced and the cells was treated with DHA $(2 \mu \mathrm{g} / \mathrm{ml})$ or DMEM medium, $12 \mathrm{~h}$ later. Following $24 \mathrm{~h}$ of DHA treatment western blot analysis and wound-healing assays were performed as aforementioned.

Statistical analysis. The data are presented as the mean \pm standard deviation. SPSS v19.0 (IBM Corp.) was used to conduct all statistical analyses. An unpaired student's t test was used to compare two groups or one-way ANOVA followed by Tukey's post hoc test for multiple comparisons. $\mathrm{P}<0.05$ was considered to indicate a statistically significant difference. GraphPad Prism v8.01 (GraphPad Software, Inc.) was used to produce the figures. 
A

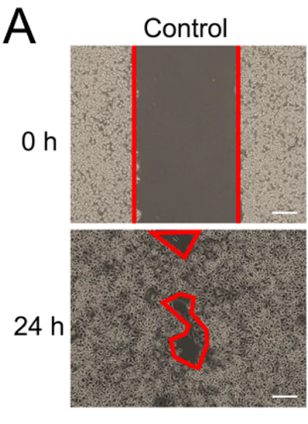

DHA $2 \mu \mathrm{g} / \mathrm{ml}$
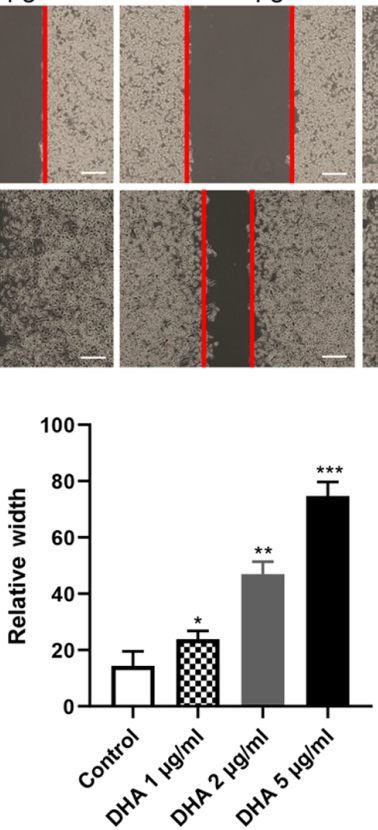

DHA $5 \mu \mathrm{g} / \mathrm{ml}$

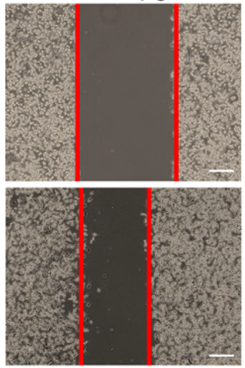

B

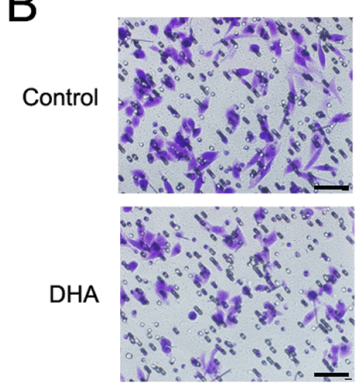

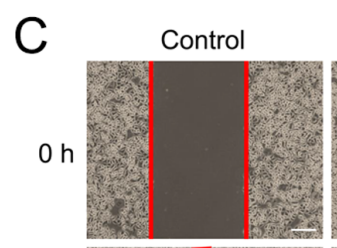

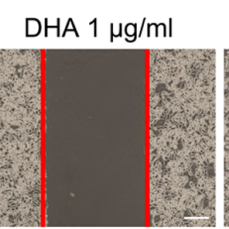

$\mathrm{DHA} 2 \mu \mathrm{g} / \mathrm{ml}$

DHA $5 \mu \mathrm{g} / \mathrm{ml}$
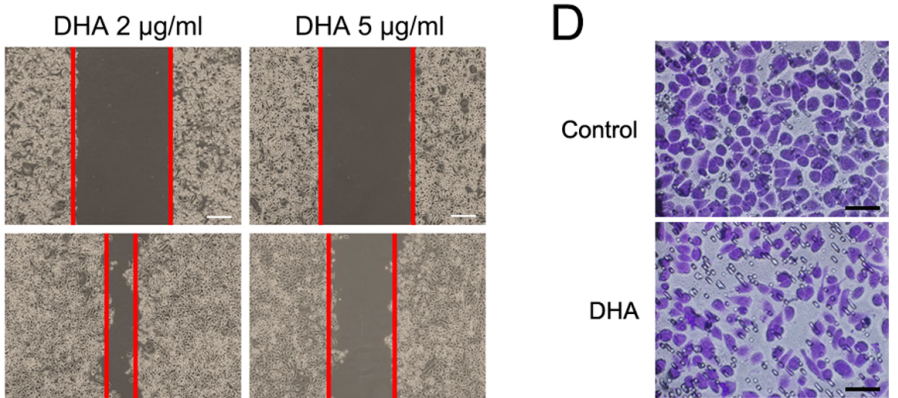

$24 \mathrm{~h}$
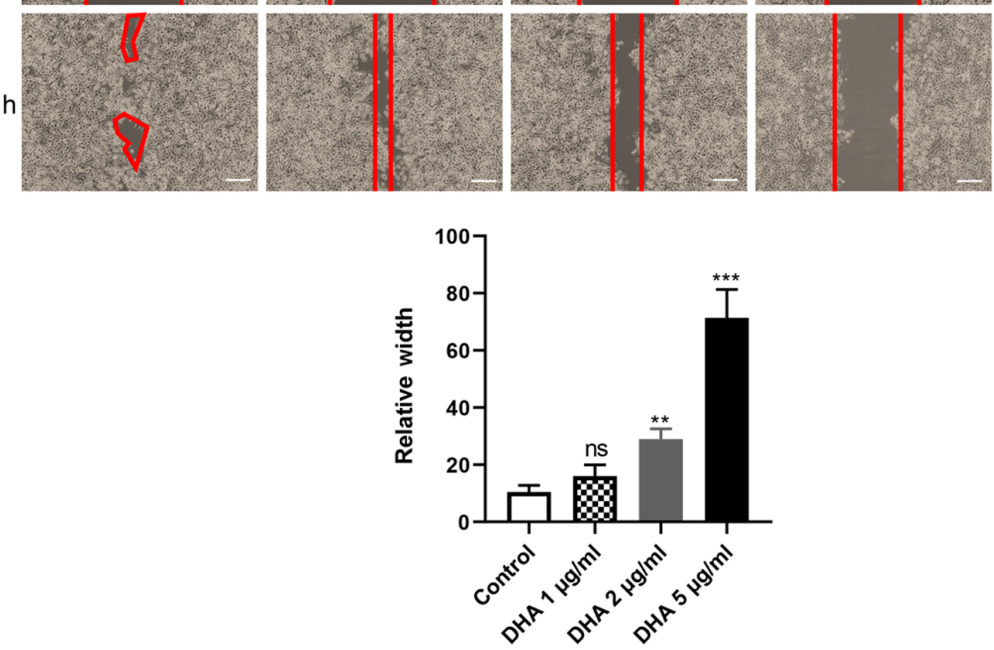

Figure 1. DHA inhibits esophageal cancer cell migration in a concentration-dependent manner. (A) A total of 1x106 Eca-109 cells were seeded into 6-well plates and incubated overnight, following which the monolayers were scratched with 1-ml tips and the cells were cultured with media containing $3 \%$ FBS, and treated with 1,2 or $5 \mu \mathrm{g} / \mathrm{ml}$ DHA for $24 \mathrm{~h}$. Images of wound closure distances were captured 0 and $24 \mathrm{~h}$ post-treatment. Scale bar, $100 \mu \mathrm{m}$. (B) $2 \times 10^{4}$ Eca- 109 cells were inoculated into Transwell chambers with serum-free medium, treated with $2 \mu \mathrm{g} / \mathrm{ml}$ DHA for $24 \mathrm{~h}$, and then fixed and stained. Migration ability was measured using the mean number of cells in 20 visual fields. Scale bar, $50 \mu \mathrm{m}$. (C) A total of $1 \times 10^{6} \mathrm{TE}-1$ cells were seeded into 6 -well plates and incubated overnight, the description of wound healing assay was similar as above. Scale bar, $100 \mu \mathrm{m}$. (D) $2 \times 10^{4} \mathrm{TE}-1$ cells were inoculated into Transwell chambers with serum-free medium and performed as above. Migration ability was measured using the mean number of cells in 20 visual fields. Scale bar, $50 \mu \mathrm{m}$. Data are represented as the mean \pm standard deviation from 3 independent experiments. ${ }^{*} \mathrm{P}<0.05,{ }^{* *} \mathrm{P}<0.01$ and ${ }^{* * * *} \mathrm{P}<0.001$ vs. control. DHA, dihydroartemisinin.

\section{Results}

DHA inhibits the migration of EC cells. To understand the effect of DHA on tumor invasion and metastasis, a cell migration assay was performed. The migratory capacity of Eca-109 and TE-1 EC cells was markedly inhibited by DHA.
DHA-treated cells possessed a markedly larger wound width compared with those of the control group, and the width of the wound was concentration-dependent (Fig. 1A and C). For the Transwell assays, the number of migratory cells in the DHA group was significantly decreased in both Eca109 $(\mathrm{P}=0.006)$ and TE-1 ( $\mathrm{P}=0.003$ ) cells (Fig. 1B and $\mathrm{D}$ ), which was consistent 
A
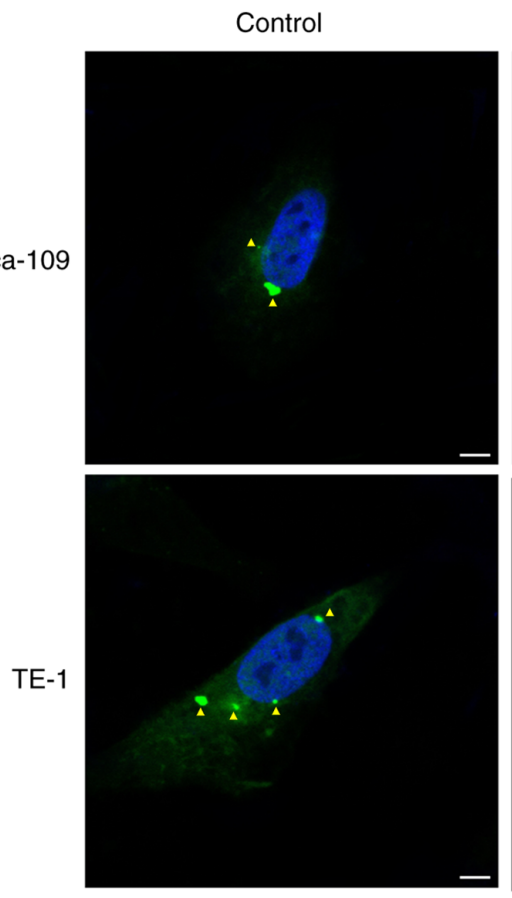

B

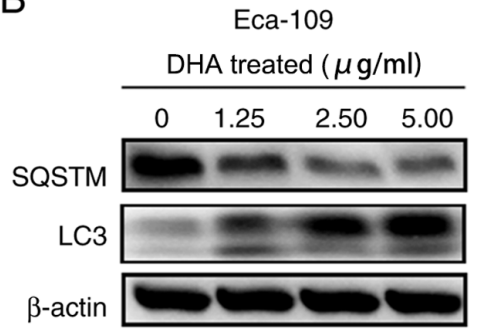

C

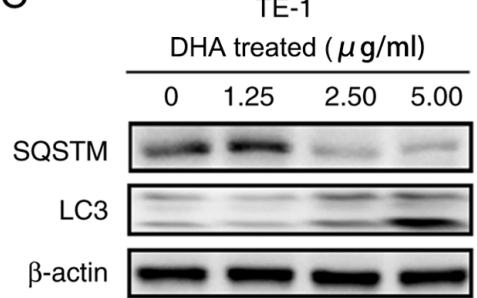

DHA
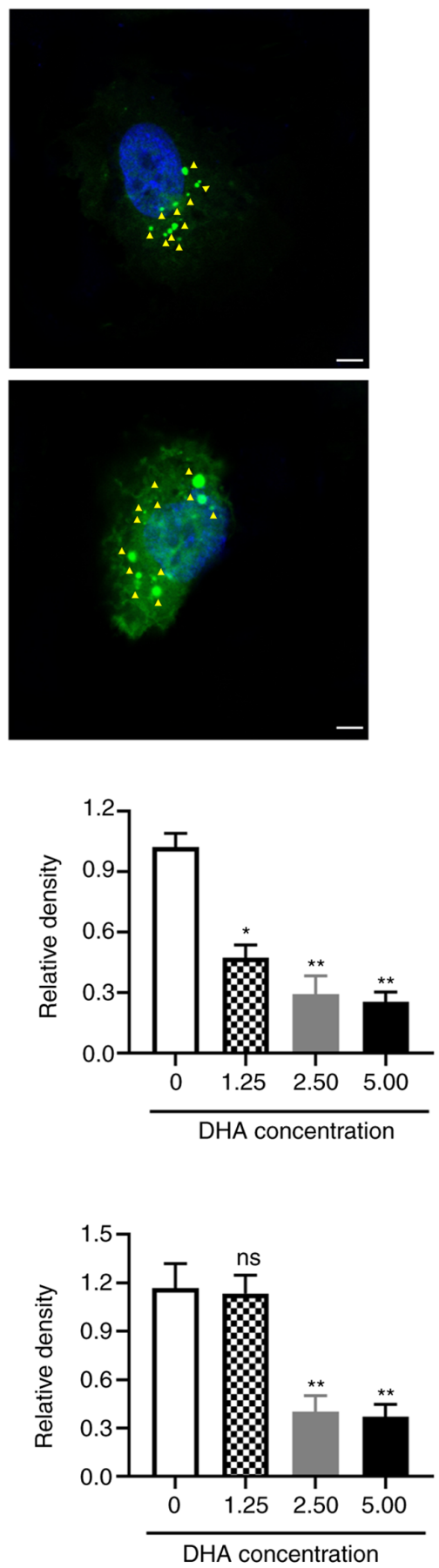
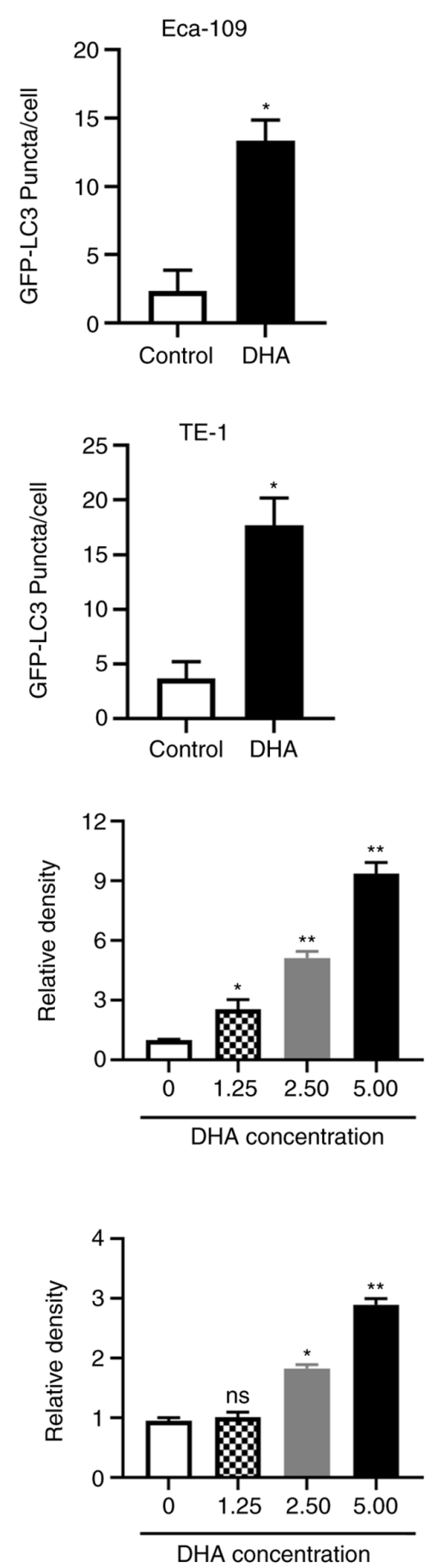

Figure 2. DHA induces autophagy in TE-1 and Eca-109 cells. (A) A total of 1x10 cells were seeded into a 24 -well plates and incubated overnight, and then transfected with an GFP-LC3 expression plasmid for $6 \mathrm{~h}$. The cells were treated with $2 \mu \mathrm{g} / \mathrm{ml}$ DHA for $24 \mathrm{~h}$, following which the cells were fixed and stained with DAPI. LC3 expression was analyzed using fluorescence microscopy, and the number of GFP-LC3 puncta per cell was determined using Image J. Yellow arrows indicate LC3 protein. Scale bar, $5 \mu \mathrm{m}$. A total of $1 \times 10^{6}$ (B) Eca-109 and (C) TE-1 cells were seeded into 6-well plates and incubated overnight, and then treated with $2 \mu \mathrm{g} / \mathrm{ml} \mathrm{DHA}$ for $24 \mathrm{~h}$. Levels of autophagy-associated proteins were detected using western blot analysis, and the relative density was determined using ImageJ software. Data are presented as the mean \pm standard deviation of 3 independent experiments. ${ }^{*} \mathrm{P}<0.05$ and ${ }^{* *} \mathrm{P}<0.01$ vs. respective control. DHA, dihydroartemisinin; GFP, green fluorescent protein; LC3, microtubule-associated protein 1A/1B-light chain 3; SQSTM, sequestosome 1; ns, not significant.

with the results of the wound healing assay. These findings demonstrate that DHA can inhibit the migration of EC cells in a concentration-dependent manner.

DHA activates autophagy in TE-1 and Eca109 cells. To investigate the underlying mechanism by which DHA inhibits the migration of EC cells, its effects on the levels of proteins associated with autophagy, a crucial process for tumor cell survival under starvation and stress (13), were investigated. EC cells were transfected with a GFP-LC3 plasmid, and the puncta of LC3 were markedly increased following DHA treatment in both TE-1 ( $\mathrm{P}=0.039)$ and Eca109 $(\mathrm{P}=0.012)$ cells (Fig. 2A). Western blot analysis also revealed a significant increase in LC3 protein expression, and a significant decrease in the 
A

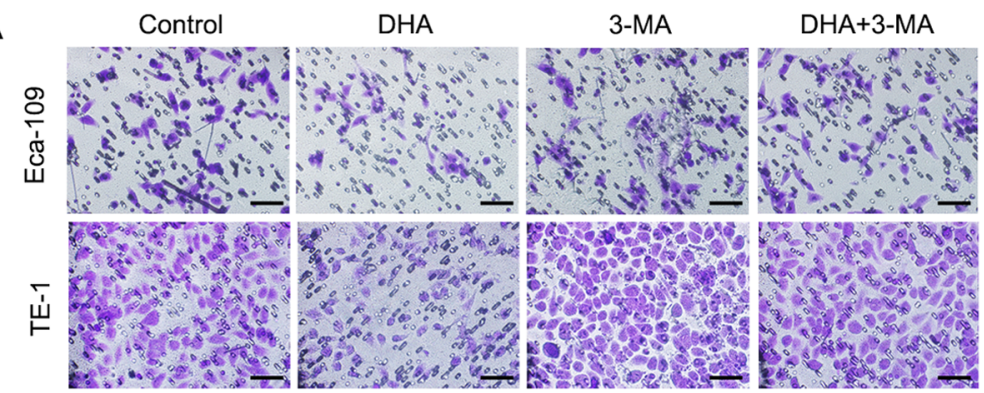

B

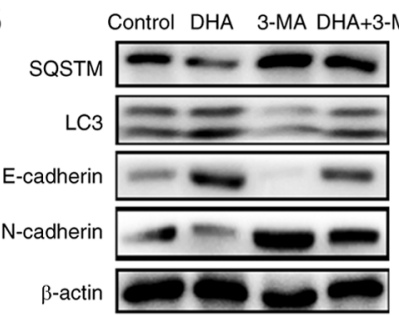

C

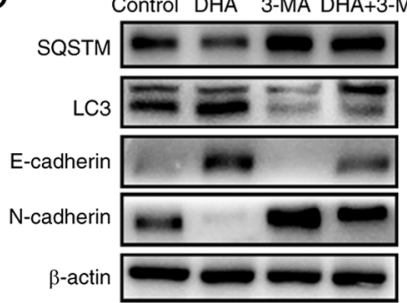

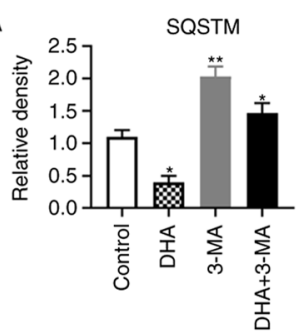
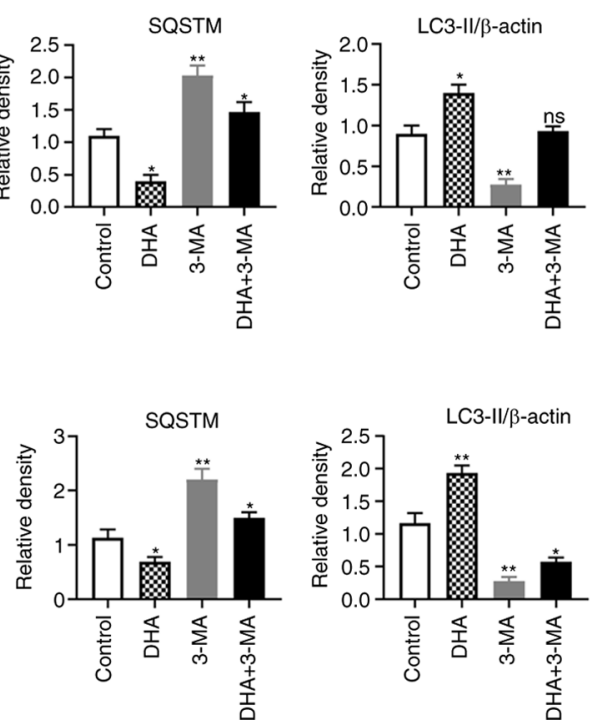
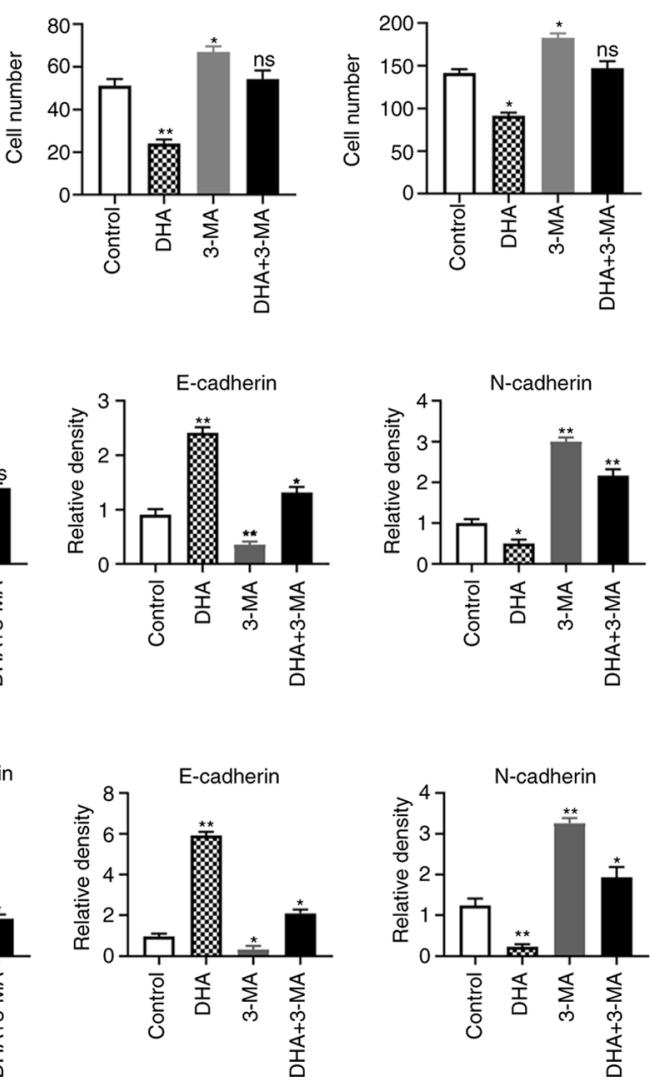

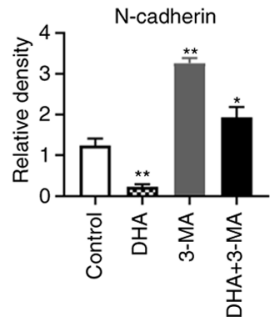

Figure 3. DHA inhibits EC cell migration by activating autophagy. (A) A total of $2 \times 10^{4}$ EC cells were inoculated into Transwell chambers with serum-free medium and treated with $2 \mu \mathrm{g} / \mathrm{ml} \mathrm{DHA,} 2 \mathrm{mmol} / 13$-MA or a combination of both for $24 \mathrm{~h}$. Membranes were then fixed and stained. Migration was recorded as the mean number of cells in 20 visual fields. Scale bar, $50 \mu \mathrm{m}$. The levels of autophagy-associated proteins in (B) Eca-109 and (C) TE-1 cells were detected using western blot analysis, and the relative density was determined using ImageJ software. Data are presented as the mean \pm standard deviation of 3 independent experiments. ${ }^{*} \mathrm{P}<0.05$ and $^{* *} \mathrm{P}<0.01$. DHA, dihydroartemisinin; EC, esophageal cancer; 3-MA, 3-methyladenine; LC3, microtubule-associated protein 1A/1B-light chain 3; SQSTM, sequestosome 1.

protein levels of SQSTM, with increasing concentrations of DHA in both cell lines (Fig. 2B and C). These results demonstrate that DHA induces autophagy in EC cells.

DHA inhibits the migration of EC cells by inducing autophagy. To determine whether DHA inhibited cell migration by activating autophagy, Transwell assays and western blot analysis were used to detect the migratory capacity and the levels of autophagy-associated proteins following treatment with the autophagy inhibitor 3-MA. In the Transwell assay, the cells of Eca-109 $(\mathrm{P}=0.007)$ and TE-1 $(\mathrm{P}=0.03)$ on the underside of the filter membrane were significantly lower in number compared with that in the control group, following treatment with DHA (Fig. 3A). However, this phenomenon was reversed, and the cell number significantly increased with co-treatment of 3-MA and DHA in both Eca-109 $(\mathrm{P}=0.003)$ and TE-1 $(\mathrm{P}=0.04)$ cells (Fig. 3A). These results indicate that the DHA-induced suppression of cell migration was weakened when autophagy was inhibited. The expression levels of the EMT markers and autophagy-associated proteins were also markedly altered; the protein levels of LC3 II and E-cadherin were significantly increased and SQSTM and N-cadherin levels decreased after DHA treatment alone. However, the expression levels of E-cadherin and LC3 II were decreased, while SQSTM and $\mathrm{N}$-cadherin were increased following co-treatment with 3-MA and DHA (Fig. 3B and C). These results indicate that DHA inhibits the migration of TE-1 and Eca-109 cells by upregulating autophagy.

The Akt/mTOR signaling pathway is involved in the DHA-induced inhibition of EC cell migration. To determine how DHA regulates autophagy and subsequently inhibits cellular migration, the expression levels of the EMT-associated proteins, E-cadherin (21), N-cadherin (22) and vimentin (23) (important components of epithelial cell loss, polarization and mesenchymal transformation) were detected. Autophagy-associated proteins, LC3 and SQSTM were also detected, as well as the phosphorylation levels of Akt and mTOR (indicating the activated forms of these proteins), which negatively regulates autophagy (24-26). As shown in Fig. 4A and B, Akt and mTOR phosphorylation were inhibited by DHA treatment, accompanied by increasing LC3 and E-cadherin expression levels, and decreasing SQSTM, vimentin and $\mathrm{N}$-cadherin expression, in both cell lines. To further determine the association between DHA and the Akt/mTOR signaling pathway, cells were transfected with CA-Akt to restore DHA-induced Akt/mTOR inhibition, and a wound-healing assay was conducted. As expected, the migratory abilities of the two EC cell lines were restored following co-treatment with DHA and CA-Akt, though migration was significantly 
A

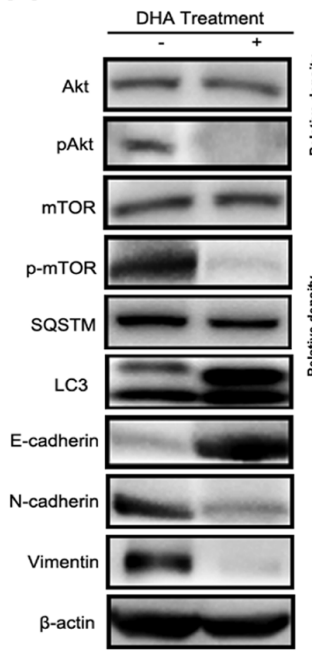

C

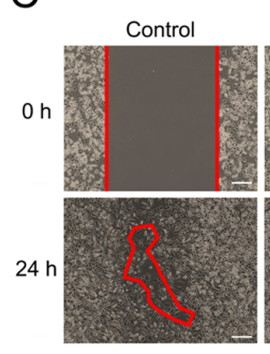

Eca-109
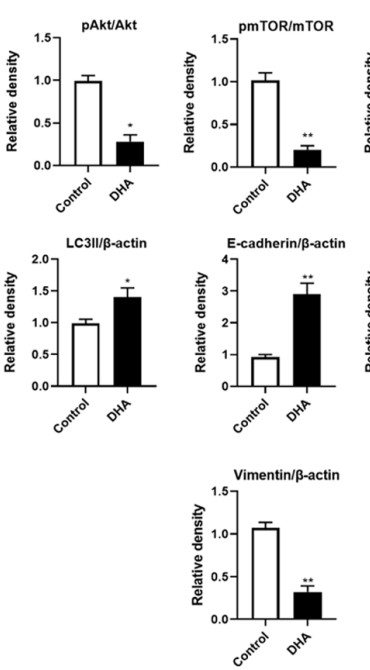

B
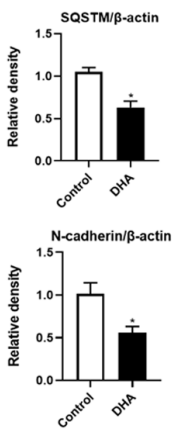
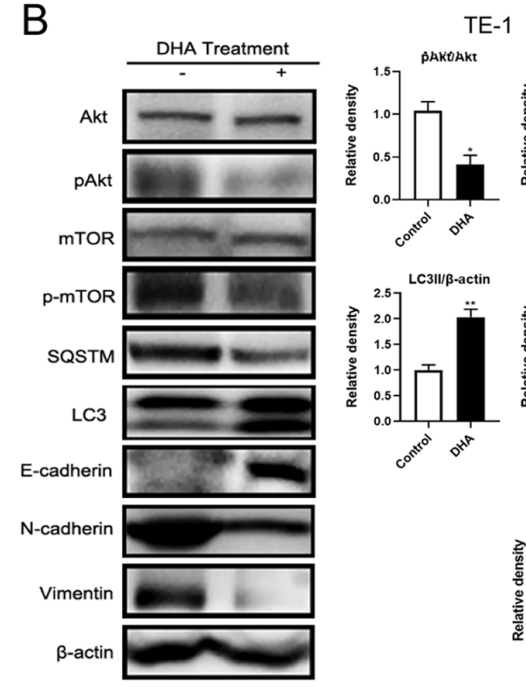

TE-1
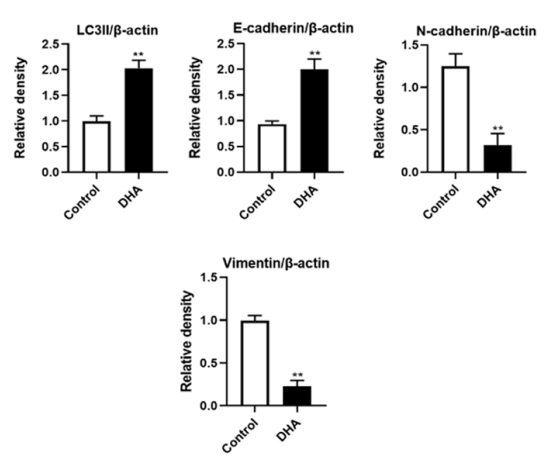

TE-1

$\mathrm{D}$

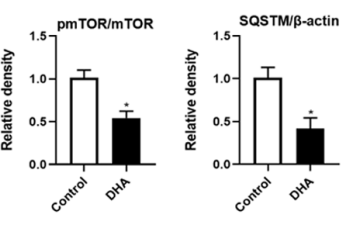

Eca-109
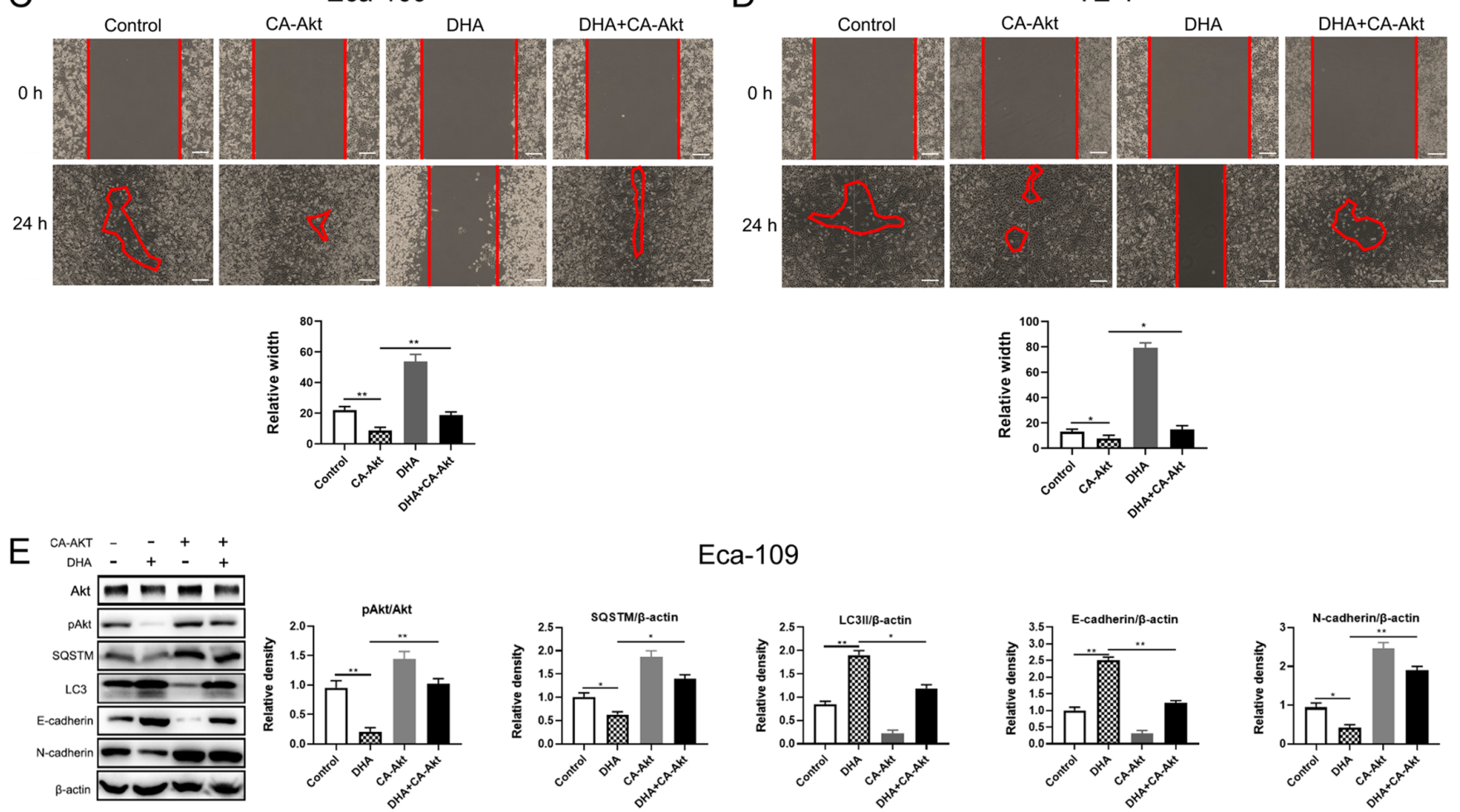

Eca-109
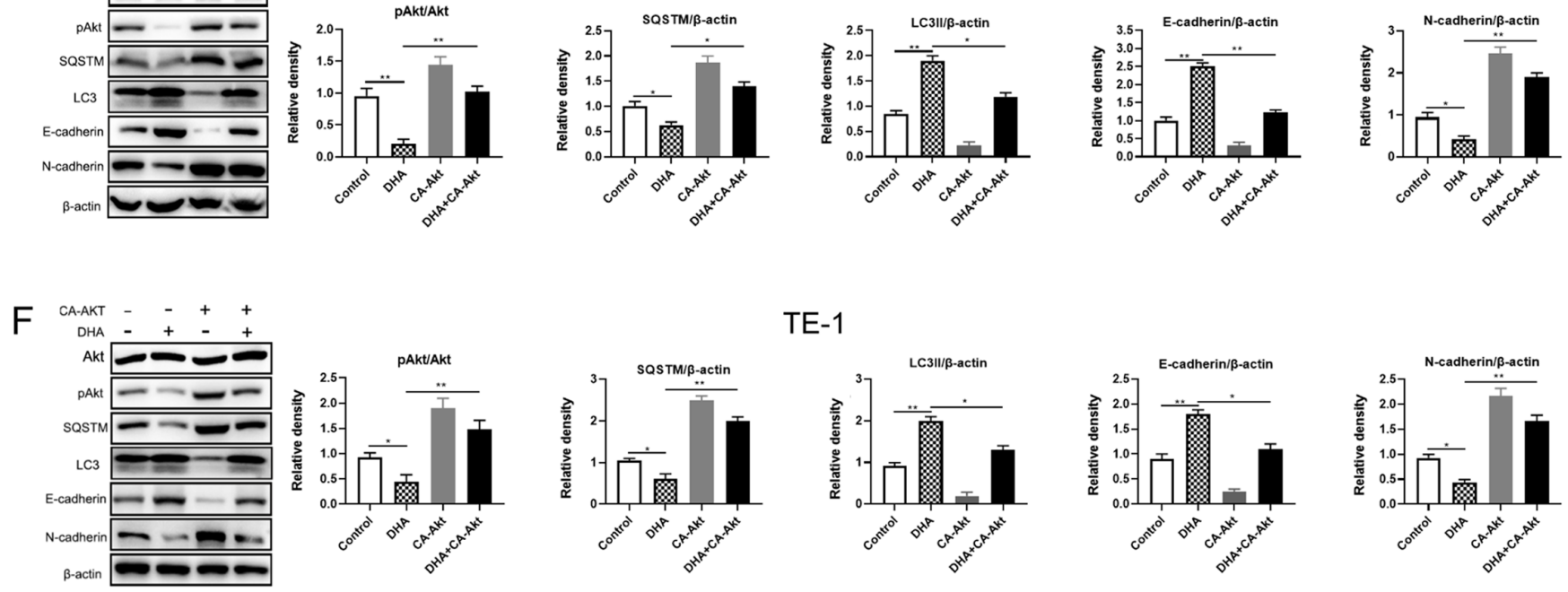

TE-1

Figure 4. DHA induces autophagy via the Akt-mTOR signaling pathway. A total of $1 \times 10^{6}$ (A) Eca-109 and (B) TE-1 cells were seeded into 6-well plates to adhere overnight, and then treated with $2 \mu \mathrm{g} / \mathrm{ml} \mathrm{DHA}$ for $24 \mathrm{~h}$. Levels of autophagy- and EMT-associated proteins were detected using western blot analysis and the relative density was analyzed using ImageJ software. Furthermore, $1 \times 10^{6}$ (C) Eca-109 and (D) TE-1 cells were seeded into 6-well plates overnight and then transfected with CA-Akt. The cell monolayers were scratched with 1-ml tips after $12 \mathrm{~h}$, subsequently cultured with medium containing $3 \%$ FBS, and then treated with $2 \mu \mathrm{g} / \mathrm{ml}$ DHA. Images of wound closure distances were captured after DHA treatment at 0 and $24 \mathrm{~h}$. Scale bar, $100 \mu \mathrm{m}$. Following $12 \mathrm{~h}$ of transfection with CA-Akt, (E) Eca-109 and (F) TE-1 cells were treated with $2 \mu \mathrm{g} / \mathrm{ml}$ DHA for $24 \mathrm{~h}$, and the levels of autophagy- and EMT-associated proteins were determined using western blot analysis and the relative densities were analyzed using ImageJ software. Data are presented as the mean \pm standard deviation of 3 independent experiments. ${ }^{*} \mathrm{P}<0.05$ and ${ }^{* *} \mathrm{P}<0.01$. DHA, dihydroartemisinin; p, phosphorylated, LC3, microtubule-associated protein 1A/1B-light chain 3; SQSTM, sequestosome 1; CA, constitutively active; EMT, epithelial-mesenchymal transition; -, without; +, with. 
decreased by DHA treatment alone (Fig. 4C and D). The results of western blot analysis were consistent with those of the wound-healing assay; in cells transfected with CA-Akt and treated with DHA, the protein levels of p-Akt, SQSTM and $\mathrm{N}$-cadherin were significantly increased compared with DHA treatment alone (Fig. 4E and F). These data indicate that DHA activates autophagy by inhibiting the phosphorylation of Akt, and subsequently suppressing the migration of TE-1 and Eca-109 cells.

\section{Discussion}

DHA, one of the primary derivatives of artemisinin, has well established anti-malarial effects and has recently been reported as a potential antitumor compound (27). An increasing amount of evidence has suggested that DHA can inhibit proliferation by promoting cell cycle arrest and apoptosis in human hepatic carcinoma (28), cholangiocarcinoma (29), cervical cancer (30) and tongue squamous carcinoma cells (31). Moreover, DHA can also prevent the migration of malignant tumor cells, such as lung cancer (32) and ovarian carcinoma cells (33). The antitumor activity of DHA in EC cells was also been shown to affect apoptosis, the cell cycle and glycolysis (29,34-38); however, to the best of our knowledge, its inhibitory effects on cell migration have not previously been reported.

EC is a common malignancy of the digestive tract, which ranks 7 th among 36 cancer types in occurrence worldwide in 2018. The prognosis of patients with EC is poor following esophagectomy, due to the metastasis and invasion of EC cells (8). EMT is a precursor of tumor metastasis, which comprises in the loss of cell polarity and the acquiring of mesenchymal cell characteristics (39). In the present study, DHA was shown to markedly inhibit EC cell migration, with reduced $\mathrm{N}$-cadherin and increased $\mathrm{E}$-cadherin protein expression. Wound-healing and Transwell assays consistently demonstrated that the migration of TE-1 and Eca-109 cells was inhibited following DHA treatment. Further investigations into the underlying mechanism of DHA revealed that migration inhibition was mediated by autophagy, and this was reversed following co-treatment with DHA and the autophagy inhibitor, 3-MA.

As a 'self-eating' process, autophagy serves a crucial role in cell survival via the degradation of aging organelles or misfolded proteins (40). An increasing number of studies have demonstrated that autophagy is associated with EMT $(41,42)$; however, the means by which autophagy regulates EMT are not completely clear. Li et al (43) found that autophagy was induced in hepatoma cells treated with serum-free Hank's medium for $6 \mathrm{~h}$, and that the expression of EMT and mesenchymal markers were decreased and increased, respectively. On the other hand, Park et al (44) demonstrated that SQSTM formed large aggresome-like induced structures (ALISs). Ubiquitination protease aggregates are generally rapidly degraded, whereas proteins bound by ALISs have a longer half-life. Transcription factors mediating EMT can also be protected in this way $(45,46)$. In the present study, DHA induced autophagy, subsequently suppressing EMT and cellular migration via the Akt/mTOR signaling pathway. DHA treatment in TE-1 and Eca-109 cells also inhibited the phosphorylation of Akt. In addition, the levels of p-Akt were significantly increased in the two cell lines, in cells transfected with a constitutively active form of Akt and active Akt restored the decreased expression level of SQSTM in DHA-treated cells. However, the underlying regulation mechanisms of DHA on AKT/mTOR pathway remains unclear, elucidating the molecular mechanisms of these processes requires further investigation.

This study was aimed to investigate the antitumor activity of DHA in esophagus cancer cells. The results demonstrated that DHA could inhibit the migration capacity of Eca109 and TE-1 cells through inducing autophagy. Exploring the underling mechanism provides new perspectives for clinical cancer therapy. However, some limitations remain in the present study. The cells were cultured with medium containing $3 \%$ FBS to maintain cell survival rather than without FBS in wound healing assay, which may influence the accuracy of this result. Furthermore, the detailed molecular mechanisms between DHA and AKT/mTOR pathway are still unknown.

In conclusion, the results of the present study demonstrated that DHA inhibits the migration of EC cells by inducing Akt/mTOR axis-mediated cytostatic autophagy. These findings may provide novel insights into the migration inhibiting activity of DHA and provide evidence to support the potential use of DHA in the clinical treatment of EC.

\section{Acknowledgements}

Not applicable.

\section{Funding}

No funding was received.

\section{Availability of data and materials}

All data generated or analyzed in this study are included in this published article.

\section{Authors' contributions}

YH conceived and designed the study. XC and LYH prepared the experimental materials and equipment. XC, LYH and SL performed the experiments and acquired the data. $\mathrm{XC}$ and LYH analyzed and interpreted the data. XC, LYH and SL drafted the manuscript and revised it critically for important intellectual content. SL and YH summarized and analyzed the final data for paper. All authors read and approved the final manuscript.

\section{Ethics approval and consent to participate}

Not applicable.

\section{Patient consent for publication}

Not applicable.

\section{Competing interests}

The authors declare that they have no competing interests. 


\section{References}

1. Bray F, Ferlay J, Soerjomataram I, Siegel RL, Torre LA and Jemal A: Global cancer statistics 2018: GLOBOCAN estimates of incidence and mortality worldwide for 36 cancers in 185 countries. CA Cancer J Clin 68: 394-424, 2018.

2. Arnold M, Soerjomataram I, Ferlay J and Forman D: Global incidence of oesophageal cancer by histological subtype in 2012 . GUT 64: 381-387, 2015.

3. Abbasi BA, Iqbal J, Ahmad R, Bibi S, Mahmood T, Kanwal S, Bashir S, Gul F and Hameed S: Potential phytochemicals in the prevention and treatment of esophagus cancer: A green therapeutic approach. Pharmacol Rep 71: 644-652, 2019.

4. Zhang Y: Epidemiology of esophageal cancer. World J Gastroenterol 19: 5598-5606, 2013.

5. Napier KJ, Scheerer M and Misra S: Esophageal cancer: A Review of epidemiology, pathogenesis, staging workup and treatment modalities. World J Gastrointest Oncol 6: 112-120, 2014.

6. Mir MM and Dar NA: Esophageal cancer in kashmir (India): An enigma for researchers. Int J Health Sci (Qassim) 3: 71-85, 2009.

7. Wong M, Hamilton W, Whiteman DC, Jiang JY, Qiao Y, Fung F, Wang $\mathrm{H}$, Chiu P, $\mathrm{Ng} \mathrm{E}$ and $\mathrm{Wu} \mathrm{J}$, et al: Global Incidence and mortality of oesophageal cancer and their correlation with socioeconomic indicators temporal patterns and trends in 41 countries. Sci Rep 8: 4522, 2018.

8. Nan L, Wei J, Jacko AM, Culley MK, Zhao J, Natarajan V, Ma H and Zhao Y: Cross-talk between lysophosphatidic acid receptor 1 and tropomyosin receptor kinase A promotes lung epithelial cel migration. Biochim Biophys Acta 1863: 229-235, 2016.

9. Tripathi V, Shin JH, Stuelten CH and Zhang YE: TGF- $\beta$-induced alternative splicing of TAK1 promotes EMT and drug resistance. Oncogene 38: 3185-3200, 2019.

10. Ota I, Masui T, Kurihara M, Yook JI, Mikami S, Kimura T, Shimada K, Konishi N, Yane K and Yamanaka T and Kitahara T: Snail-induced EMT promotes cancer stem cell-like properties in head and neck cancer cells. Oncol Rep 35: 261-266, 2016.

11. Mooney SM, Talebian V, Jolly MK, Jia D, Gromala M, Levine H and McConkey BJ: The GRHL2/ZEB feedback loop-a key axis in the regulation of EMT in breast cancer. J Cell Biochem 118 2559-2570, 2017

12. Cano A and Portillo F: An emerging role for class I bHLH E2-2 proteins in EMT regulation and tumor progression. Cell Adh Migr 4: 56-60, 2010.

13. Gao FX, Wu J and Ren DL: Effect of epithelial-to-mesenchymal transition on biological activity of NK cells in esophageal squamous cell carcinoma. Sichuan Da Xue Xue Bao Yi Xue Ban 50: 40-47, 2019 (In Chinese)

14. Yokozaki H, Koma YI, Shigeoka M and Nishio M: Cancer as a tissue: The significance of cancer-stromal interactions in the development, morphogenesis and progression of human upper digestive tract cancer. Pathol Int 68: 334-352, 2018.

15. Mladinich M, Ruan D and Chan CH: Tackling cancer stem cells via inhibition of EMT transcription factors. Stem Cells Int 2016: 5285892, 2016.

16. Pattabiraman DR, Bierie B, Kober KI, Thiru P, Krall JA, Zill C, Reinhardt F, Tam WL and Weinberg RA: Activation of PKA leads to mesenchymal-to-epithelial transition and loss of tumor-initiating ability. Science 351: d3680, 2016.

17. Gugnoni M, Sancisi V, Gandolfi G, Manzotti G, Ragazzi M Giordano D, Tamagnini I, Tigano M, Frasoldati A, Piana S and Ciarrocchi A: Cadherin-6 promotes EMT and cancer metastasis by restraining autophagy. Oncogene 36: 667-677, 2017.

18. Ouyang F, Huang H, Zhang M, Chen M, Huang H, Huang F and Zhou S: HMGB1 induces apoptosis and EMT in association with increased autophagy following $\mathrm{H} / \mathrm{R}$ injury in cardiomyocytes. Int J Mol Med 37: 679-689, 2016.

19. Lin R, Zhang Z, Chen L, Zhou Y, Zou P, Feng C, Wang L and Liang G: Dihydroartemisinin (DHA) induces ferroptosis and causes cell cycle arrest in head and neck carcinoma cells. Cancer Lett 381: 165-175, 2016.

20. Liu T, Guo J, Wang T, Zhang S, Yu X, Hou C and Guo D: Network pharmacology-based analysis of mechanisms of the anti-hepatocellular carcinoma effect by dihydroartemisinin. Discov Med 28: 139-147, 2019.

21. Daugaard I, Sanders KJ, Idica A, Vittayarukskul K, Hamdorf M, Krog JD, Chow R, Jury D, Hansen LL, Hager H, et al: miR-151a induces partial EMT by regulating E-cadherin in NSCLC cells. Oncogenesis 6: e366, 2017.
22. Zhang X, Liu G, Kang Y, Dong Z, Qian Q and Ma X: N-cadherin expression is associated with acquisition of EMT phenotype and with enhanced invasion in erlotinib-resistant lung cancer cell lines. PLoS One 8: e57692, 2013.

23. Ivaska J: Vimentin: Central hub in EMT induction? Small GTPases 2: 51-53, 2011

24. Liang P, Jiang B, Li Y, Liu Z, Zhang P, Zhang M, Huang X and Xiao X: Autophagy promotes angiogenesis via AMPK/Akt/mTOR signaling during the recovery of heat-denatured endothelial cells. Cell Death Dis 9: 1152, 2018.

25. Yang J, Pi C and Wang G: Inhibition of $\mathrm{PI} 3 \mathrm{~K} / \mathrm{Akt} / \mathrm{mTOR}$ pathway by apigenin induces apoptosis and autophagy in hepatocellular carcinoma cells. Biomed Pharmacother 103: 699-707, 2018.

26. Fan S, Zhang B, Luan P, Gu B, Wan Q, Huang X, Liao W and Liu J: PI3K/AKT/mTOR/p70S6K pathway is involved in A 325 -35-induced autophagy. Biomed Res Int 2015: 161020, 2015.

27. Cao P, Leng D, Li Y, Zhang Z, Liu L and Li X: Progress on anti-tumor molecular mechanisms of dihydroartemisinin. Zhejiang Da Xue Xue Bao Yi Xue Ban 45: 501-507, 2016 (In Chinese).

28. Zhang CZ, Zhang H, Yun J, Chen GG and Lai PB: Dihydroartemisinin exhibits antitumor activity toward hepatocellular carcinoma in vitro and in vivo. Biochem Pharmacol 83: 1278-1289, 2012

29. Thongchot S, Vidoni C, Ferraresi A, Loilome W, Yongvanit P, Namwat $\mathrm{N}$ and Isidoro $\mathrm{C}$ : Dihydroartemisinin induces apoptosis and autophagy-dependent cell death in cholangiocarcinoma through a DAPK1-BECLIN1 pathway. Mol Carcinog 57: 1735-1750, 2018.

30. Li X, Ba Q, Liu Y, Yue Q, Chen P, Li J, Zhang H, Ying H, Ding $\mathrm{Q}$, Song $\mathrm{H}$, et al: Dihydroartemisinin selectively inhibits PDGFRalpha-positive ovarian cancer growth and metastasis through inducing degradation of PDGFRalpha protein. Cell Discov 3: 17042, 2017.

31. Shi X, Wang L, Li X, Bai J, Li J, Li S, Wang Z and Zhou M: Dihydroartemisinin induces autophagy-dependent death in human tongue squamous cell carcinoma cells through DNA double-strand break-mediated oxidative stress. Oncotarget 8: 45981-45993, 2017.

32. Jiang J, Geng G, Yu X, Liu H, Gao J, An H, Cai C, Li N, Shen D, Wu X, et al: Repurposing the anti-malarial drug dihydroartemisinin suppresses metastasis of non-small-cell lung cancer via inhibiting NF-kappaB/GLUT1 axis. Oncotarget 7: 87271-87283, 2016.

33. Im E, Yeo C, Lee HJ and Lee EO: Dihydroartemisinin induced caspase-dependent apoptosis through inhibiting the specificity protein 1 pathway in hepatocellular carcinoma SK-Hep-1 cells. Life Sci 192: 286-292, 2018.

34. Li Y, Sui H, Jiang C, Li S, Han Y, Huang P, Du X, Du J and Bai Y: Dihydroartemisinin increases the sensitivity of photodynamic therapy via NF- $\kappa \mathrm{B} / \mathrm{HIF}-1 \alpha / \mathrm{VEGF}$ pathway in esophageal cancer cell in vitro and in vivo. Cell Physiol Biochem 48: 2035-2045, 2018.

35. Jiang C, Li S, Li Y and Bai Y: Anticancer effects of dihydroartemisinin on human esophageal cancer cells in vivo. Anal Cell Pathol (Amst) 2018: 8759745, 2018.

36. Li S, Huang P, Gan J, Ling X, Du X, Liao Y, Li L, Meng Y, Li Y and Bai Y: Dihydroartemisinin represses esophageal cancer glycolysis by down-regulating pyruvate kinase M2. Eur J Pharmacol 854: 232-239, 2019.

37. Li YJ, Zhou JH, Du XX, Jia DX, Wu CL, Huang P, Han Y, Sui H, Wei XL, Liu L, et al: Dihydroartemisinin accentuates the anti-tumor effects of photodynamic therapy via inactivation of NF- $\mathrm{KB}$ in Eca109 and Ec9706 esophageal cancer cells. Cell Physiol Biochem 33: 1527-1536, 2014.

38. Du XX, Li YJ, Wu CL, Zhou JH, Han Y, Sui H, Wei XL, Liu L, Huang P, Yuan HH, et al: Initiation of apoptosis, cell cycle arrest and autophagy of esophageal cancer cells by dihydroartemisinin. Biomed Pharmacother 67: 417-424, 2013.

39. Alonso-Alconada L, Eritja N, Muinelo-Romay L, Barbazan J, Lopez-Lopez R, Matias-Guiu X, Gil-Moreno A, Dolcet X and Abal M: ETV5 transcription program links BDNF and promotion of EMT at invasive front of endometrial carcinomas. Carcinogenesis 35: 2679-2686, 2014

40. Zhou S, Zhao L, Kuang M, Zhang B, Liang Z, Yi T, Wei Y and Zhao X: Autophagy in tumorigenesis and cancer therapy: Dr. Jekyll or Mr. Hyde? Cancer Lett 323: 115-127, 2012.

41. Colella B, Faienza F and Di Bartolomeo S: EMT regulation by autophagy: A new perspective in glioblastoma biology. Cancers (Basel) 11: 312, 2019. 
42. Feng H, Zhao X, Guo Q, Feng Y, Ma M, Guo W, Dong X, Deng C, Li C, Song X, et al: Autophagy resists EMT process to maintain retinal pigment epithelium homeostasis. Int J Biol Sci 15: 507-521, 2019.

43. Li J, Yang B, Zhou Q, Wu Y, Shang D, Guo Y, Song Z, Zheng Q and Xiong J: Autophagy promotes hepatocellular carcinoma cell invasion through activation of epithelial-mesenchymal transition. Carcinogenesis 34: 1343-1351, 2013.

44. Park S, Ha SD, Coleman M, Meshkibaf S and Kim SO: p62/SQSTM1 enhances NOD2-mediated signaling and cytokine production through stabilizing NOD2 oligomerization. PLoS One 8: e57138, 2013.
45. Qiang L, Zhao B, Ming M, Wang N,He TC, Hwang S, Thorburn A and He YY: Regulation of cell proliferation and migration by p62 through stabilization of Twist1. Proc Natl Acad Sci USA 111: 9241-9246, 2014

46. Qiang L and He YY: Autophagy deficiency stabilizes TWIST1 to promote epithelial-mesenchymal transition. Autophagy 10: 1864-1865, 2014

(i) $\odot$ This work is licensed under a Creative Commons Attribution-NonCommercial-NoDerivatives 4.0 International (CC BY-NC-ND 4.0) License. 